\title{
Sustainable and functional tandem repeat protein fibers and films
}

Microfibers are one of the most common forms of plastic debris in aquatic habitats. To address this emerging threat, we focus on primary drivers of plastic pollution, as well as potential alternatives to synthetic plastics. First, technological developments since the cognitive revolution and their consequences for human population expansion will be introduced, followed by an examination of the history and technological drivers of plastics production. Finally, recent advances in biotechnology and materials science for biosynthesis of environmentally sustainable materials will be discussed. For example, discoveries in nanotechnology combined with parallel improvements in biotechnology and synthetic biology demonstrated that sustainable fibers, which are potential alternatives to synthetic plastics, with properties engineered precisely to optimize fiber performance could be achieved. Using this approach, functional programmable materials with user defined physical properties to address the microfiber pollution are recently created. Our approach is based on new protein based fibers screened by ultrafast microscopy technique that could help replace synthetic fibers.

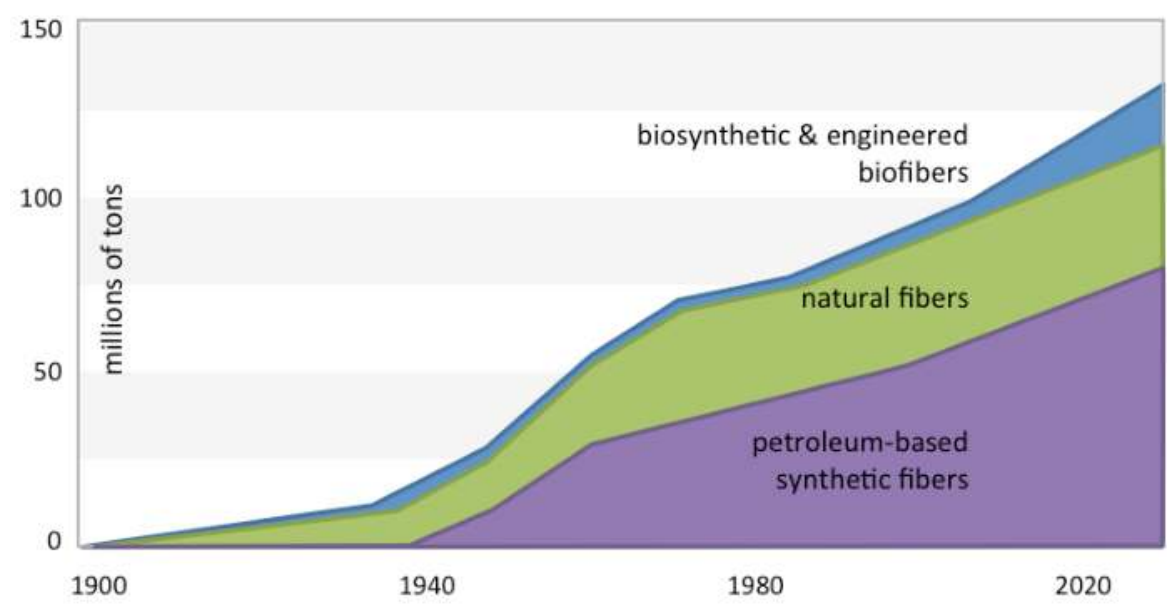

Figure 1. Timeline of synthetic, natural and engineered fibers as a function of production values 